\title{
Seismic reflections de-noising and recognition using Empirical Mode Decomposition and Continuous Wavelet Transformation
}

\author{
Amjad Ali* | Chen Sheng-Chang \\ School of Earth Sciences, Zhejiang University, Hangzhou City, Zhejiang Province, China. \\ * Corresponding Author Email: aali@zju.edu.cn
}

Received: December 8, $2021 \quad$ Accepted: December 28, $2021 \quad$ Published: January 30, 2022

\begin{abstract}
Current developments in signal processing are allowing for enhanced seismic illustrations and investigation of subsurface structures. Recently, Empirical Mode Decomposition (EMD) and Continuous Wavelet Transformation (CWT) have been introduced to extract various features from a time series dataset. In this investigation, seismic signal with $10 \%$ Gaussian noise is transformed into sub-signals by EMD analysis to improve the Signal-to-Noise Ratio (SNR). Then, CWT is implemented for each sub-signal to identify the exact locations of seismic reflections. The main objective of this study is to utilize the EMD as a noise filter in the timedomain and CWT to recognize the anomalous zone in each sub-signal. Based on the results of EMD and CWT, the true representation of a seismic signal with minimum noise in the time domain has been achieved. The successful integration of EMD and CWT is achieved in terms of the identification of true seismic reflections as localized anomalous zones at $0.8 \mathrm{sec}, 1 \mathrm{sec}$, and $1.07 \mathrm{sec}$.
\end{abstract}

Keywords: Empirical Mode Decomposition (EMD), Continuous Wavelet Transformation (CWT), Signal-to-Noise ratio (SNR), Gaussian noise, seismic reflection.

\section{How to Cite:}

Ali, A., \& Sheng-Chang, C. (2022). Seismic reflections de-noising and recognition using Empirical Mode Decomposition and Continuous Wavelet Transformation. Natural and Applied Sciences International Journal (NASIJ), 3(1), 1-12. https://doi.org/10.47264/idea.nasij/3.1.1

\section{Publisher's Note:}

IDEA Publishers Group stands neutral regarding jurisdictional claims in the published maps and institutional affiliations.

Copyright: ( 2022 The Author(s), published by IDEA Publishers Group.

\section{Licensing:}

This is an Open Access article published under the Creative Commons Attribution-NonCommercial 4.0 International License (http://creativecommons.org/licenses/by-nc/4.0/) 


\section{Introduction}

Generally, seismic data is generated by a complex framework of which we know nothing. In such a framework, predictable behaviour, for example, patterns and periodicities, is consequently of incredible interest for a geoscientist. Most conventional numerical techniques that look at periodicities in the frequency domain, for example, Fourier investigation, assumed that the underlying processes are stationary. While seismic data is non-stationary because the frequency is changing with time. Over time, various algorithms have been implemented for the characterization of the non-stationary signal. For example, Peyton et al. (1998) implemented the spectral decomposition along with coherency for the interpretation of incise valley. Later, Partyka et al. (1999) did the reservoir characterization by adopting the widowed spectral analysis. Similarly, Castagna et al. (2003) utilized the matching pursuit technique to identify low-frequency regions beneath petroleum reservoirs. While Iqbal et al. (2018a) implemented AVO analysis and rock physics computation to identify hydrocarbon reservoirs in two different case studies. Furthermore, Liu et al. (2021) proposed the green function for a seismic field to estimate the seismic signal parameters.

In this study, we tried to integrate Huang's Empirical Mode Decomposition (EMD) (Huang et $a l .$, 1998) and Continuous Wavelet Transformation (CWT) to process and interpret the geophysical non-stationary signal. EMD is a very effective time-domain filter and CWT is a good mathematical tool to localize the anomalous zone in the time domain. The main objective of this work is to illustrate the potential of these new algorithms to enhance SNR and locate the seismic reflections at their exact time domain.

\section{Geological model}

Initially, a geological model is assumed for this study. Then, a seismic trace of the geological anticline structure has been used to analyse the integration of EMD and CWT. Figure 1 represents the geological model, in the model, the $3^{\text {rd }}$ layer acts as a reservoir and it is pure sandstone with $10 \%$ porosity. The upper-most portion of the sandstone reservoir is live-oil immersed, and the lower portion is saline-water saturated. While the second layer acts as a cap rock for hydrocarbon reservoir and it is shale. Similarly, the first layer of the geological model begins from the surface to the pinnacle of the $2^{\text {nd }}$ layer. Batzle and Wang's (1992) equations are utilized to compute the elastic characteristics of two particular pore fluids (Live-oil \& saline-water). For pore-fluids substitution in sandstone reservoir, the equation of Gassmann is adopted.

Figure 1: Three-layer geological model

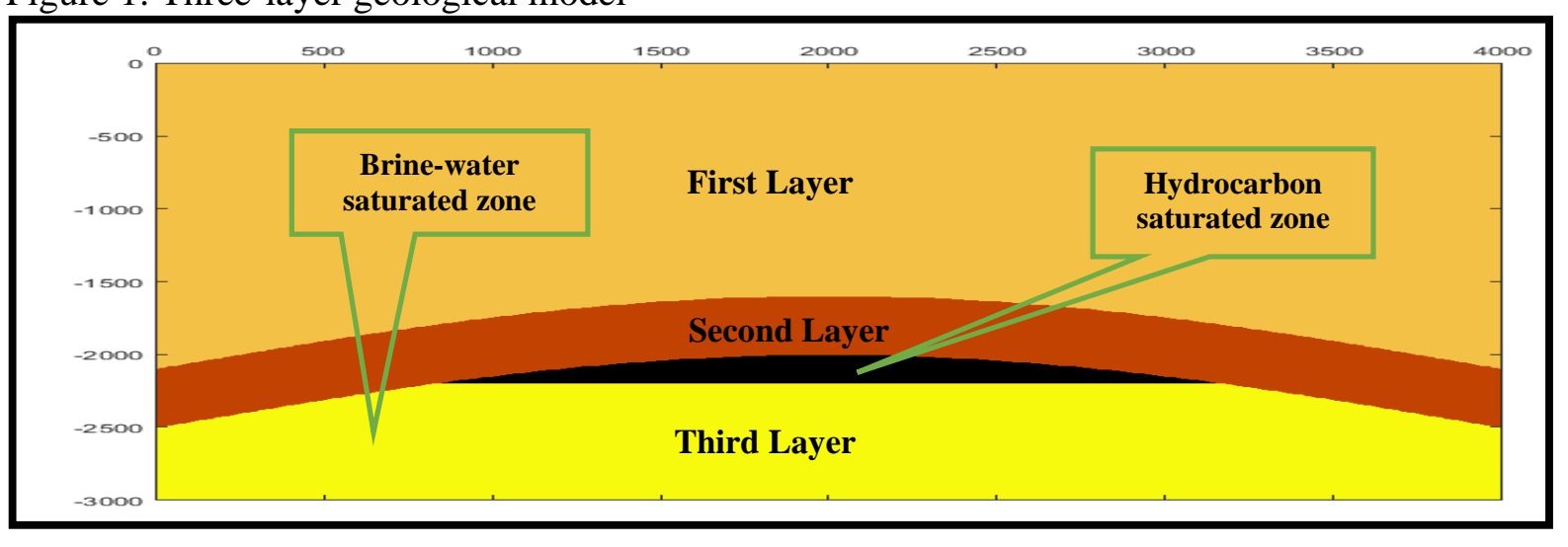

NASIJ, 2022, 3(1), 1, 1-12 
At the first stage, 1D seismic trace with $10 \%$ Gaussian-noise is generated at the centre of the geological structure utilizing the convolution model (Yilmaz, 2001), as shown in figure 2. This 1D seismic trace is later used for the implementation of EMD for de-noising. Next, CWT is implemented on each IMD of EMD to validate the results and identify each reflector at its exact location.

Figure 2: Synthetic seismic trace of the geological model with and without $10 \%$ Gaussian Noise

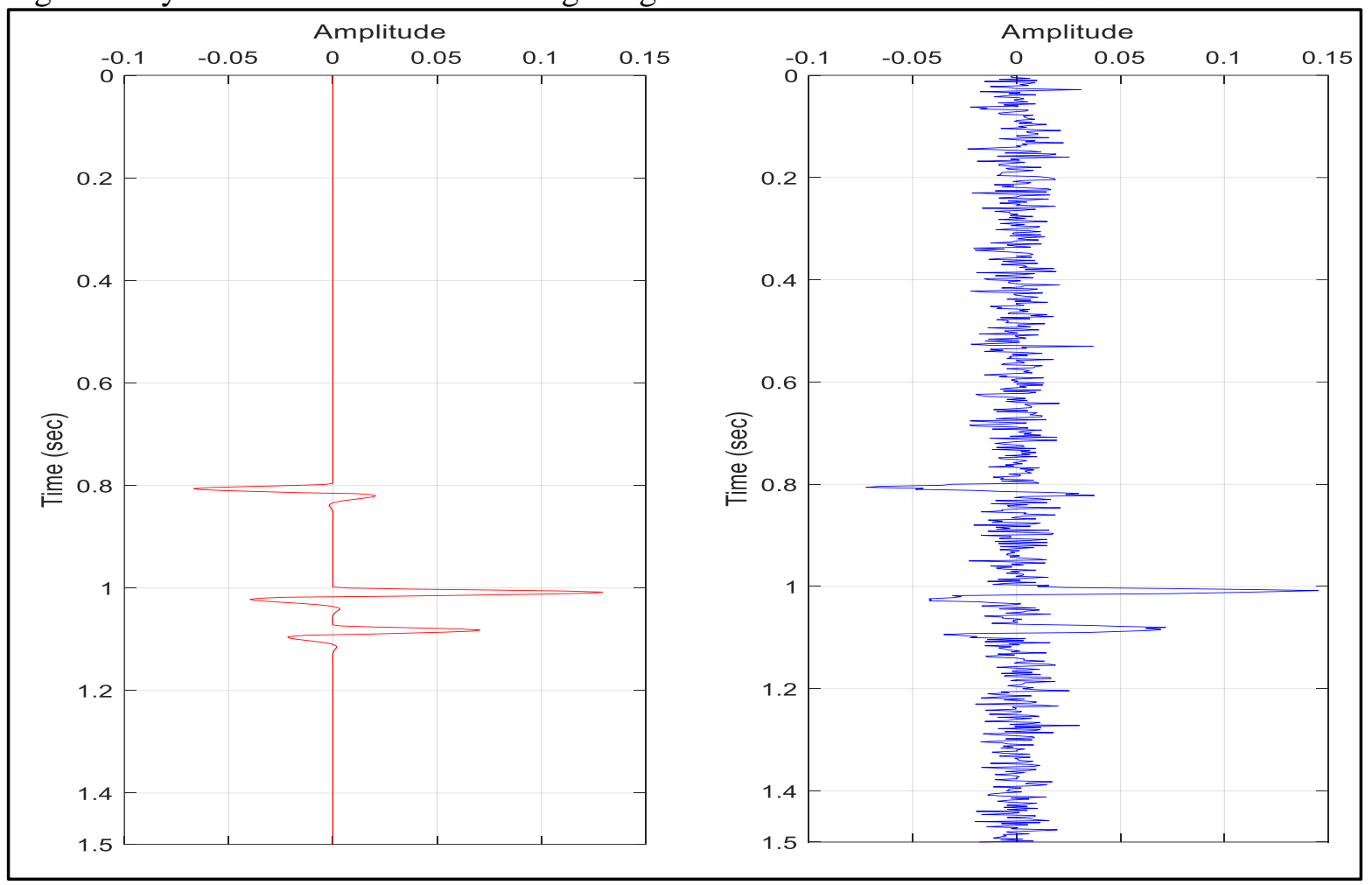

\subsection{Empirical Mode Decomposition (EMD)}

EMD obtained absolute recognition in the signal processing society, but the seismic exploration society did not give much attention to EMD. Magrin-Chagnolleau and Baraniuk (1999) illustrated that the seismic signals can be examined by utilizing the EMD. Furthermore, the Rilling et al. (2004) and Flandrin et al. (2004) studies were empowered due to the similarities among the signals being. These studies recommended the implementation of EMD as a detrending and de-noising tool, which is an essential part of seismic data processing.

EMD is a recently developed algorithm to decompose and study non-stationary time series data set (Huang et al., 1998; Wu \& Huang, 2004). The EMD has an assumption that the time series should consist of intrinsic mode oscillations. These modes or sub-signals are called Intrinsic Mode Functions (IMFs).

The main purpose of EMD is to break down a time-series signal into IMFs. EMD generates several IMFs based on the local maxima and characteristics of data, where the sum of all these IMFs is the original signal (Coughlin \& Tung, 2004). There are two basic conditions for each IMF: (1) the number of local troughs and peaks and zero-crossing have to equate, and (2) the arithmetic mean of the envelope described by the local extrema must be equal to zero (Zhongxuan et al., 2004). The procedure of the generation of IMFs exists in the concept of 
sifting given by Huang et al. (1998). The output of sifting is to get rid of all kinds of trends that forbid the IMF from satisfying the conditions of IMFs. Once the conditions of IMFs are satisfied, the EMD will not generate any more IMFs. Generally, to envelope the signal, a cubic spline is applied to every IMF. The arithmetic mean of the envelope is referred to as mean spline. This arithmetic mean of the spline is subtracted from the original signal to generate a new IMF. The procedure is reiterated on every new IMF as far as the IMF's conditions are fulfilled. This is not possible to get a zero arithmetic mean spline for a signal; therefore, the conditions should be set to gain when the sifting process has efficiently generated IMFs. This criterion is pre-arranged between two successive components of sifting for RMS tolerance. For example, $X_{o}(t)$ is sifted to produce the first IMF, $X_{1}(t)$, by subtracting the mean spline $\left(m_{o}(t)\right)$. WhileRMS is computed for $X_{o}(t), X_{1}(t)$, and their difference. According to equation1 , the sifting process carries on if the RMS contrast is more than tolerance.

$$
\begin{gathered}
\frac{1}{J}\left(\left(\sum_{j=1}^{J} X_{n-1}(t)^{2}\right)^{1 / 2}-\left(\sum_{j=1}^{J} X_{n}(t)^{2}\right)^{1 / 2}\right) \\
>\text { tolerance } \\
S_{n+1}(t)=S_{n}(t)-m_{n}(t)
\end{gathered}
$$

Here, $\mathrm{n}$ is $1,2,3$, and $\mathrm{J}$ represents the total no. of samples in the signal. Usually, $1^{\text {st }}$ IMF consists of the highest frequencies of the signal, while a no. of low-frequency components is removed. Then, the $1^{\text {st }}$ IMF will be subtracted from the original time series signal to generate the $2^{\text {nd }}$ IMF. This process of sifting and generation of IMFs keeps going unless it is terminated manually, or the process produces all the IMFs along with the residue. The equation of EMD for a time-series signal can be written as.

$$
C_{o}(t)=\left(\sum_{n=1}^{N} C_{n}(t)\right)+r(t)
$$

Here, $C_{o}(t)$ is time-series data, $C_{n}(t)$ is the output as IMFs, $\mathrm{N}$ is the no. of IMFs is generated, and $r(t)$ is the residual of the signal.

\subsection{Continuous Wavelet Transform (CWT)}

Continuous Wavelet transformation (WT) is a well-known mathematical means to analyse the localized changes within a time series dataset (Mallat \& Stephane, 1987). WT produces a timescale range instead of a time-frequency range (Rioul \& Vetterli, 1991). WT is a powerful technique to examine arbitrary oscillation in a time-series dataset (Holschneider, 1995). While WT has a sub-class, and it is called CWT. It is far generally exercised for characteristic extraction. The basic idea of CWT was obtained from Gabor's (1946) Short-Time Fourier Transform (STFT), in which he kept constant the time window to acquire the entire spectrum of frequencies within the time window.

Klausner et al. (2013) has summarized the implementation of CWT to focus on distinct troubles for example quality of the dataset, availability of data, database disparity, and inaccuracy 
factors. For the assessment of CWT, Hua et al. (2014) tested normalizations to attain an exclusive depiction of a dataset. Adhikari (2015) accomplished an analysis to characterize a time series dataset in the time and scale domain. Iqbal et al. (2018b) have effectively consolidated the CWT and Mann Kendal (MK) test to determine the silt burden as well as overflow changes in the river. Afterward, Palupi (2018) utilized CWT for gauging minerals profundities in a mineral fertile area in Java, Indonesia. For singularity and damage detection, Zhou and Chen (2020) adopted CWT for a comparative analysis between Pet-hat and Morlet wavelets.

In CWT, the mother wavelet acts as a filter for the dataset and the wavelet goes through the translation and dilation process. CWT can extend a signal within the time and frequency domain, simultaneously. In this way, CWT can analyse and locate a restricted peculiarity in a signal distributed at equivalent intervals. The mathematical representation of CWT can be formulated as.

$$
W(a, b)=\frac{1}{\sqrt{a}} \int_{-\infty}^{\infty} f(t) \frac{1}{a} \psi *\left(\frac{t-b}{a}\right) d t
$$

In equation $3, \psi(\mathrm{t})$ represents the mother wavelet and $*$ is a complex conjugate. While, $\mathrm{W}$ (a, b) are continuous wavelet transform's coefficients, here, ' $a$ ' is the translation and ' $b$ ' is dilation parameter. In CWT, the mother wavelet will get contracted because of small estimations of ' $a$ ', and subsequently, it generated high-frequency functions. The mother wavelet will get stretched because of high estimations of 'a' and delivers low-frequency functions. Since time series is time-bounded, that is the reason it gets influenced by edge artifacts and it is called the Cone of Influence (COI) (Torrence \& Compo, 1998).

In this examination, CWT is utilized to distinguish and affirm seismic reflectors as localized anomalies. To limit the peculiar regions, for the mother wavelet, the 'Haar' wavelet has been used as it has the ability to identify unexpected discontinuities and sudden variations in timeseries dataset. In CWT, two basic functions, i.e., selection of mother wavelet and scaling function perform vital parts. Haar wavelet's scaling function is characterized as follows.

$$
\emptyset(\mathrm{x})= \begin{cases}1, & \text { if } 0 \leq x<1 \\ 0, & \text { otherwise }\end{cases}
$$

Whereas the Haar wavelet is characterized as.

$$
\psi(\mathrm{x})=\left\{\begin{aligned}
1, & 0 \leq x<1 / 2 \\
-1, & 1 / 2 \leq x<1 \\
0, & \text { otherwise }
\end{aligned}\right.
$$

The investigation is presented as scalogram, 2D, and 3D CWT coefficients.

\section{Results and discussion}

In this examination, three seismic reflectors of a geological model are identified through the integration of EMD and CWT. In the geological model, the third layer serves as a reservoir, where an upper-most aspect of the reservoir is live-oil immersed, and the remaining part is 
saline water-saturated. The target of this investigation is to highlight feature extraction through the integration of EMD and CWT. At first, a 10\% Gaussian noise is included in the seismic reflection dataset to create a reasonable situation. Because of the addition of noise, it's difficult to characterize accurately various seismic reflectors in seismic data. However, EMD can disintegrate the given signal into several IMFs. After the implementation of EMD, CWT is executed to recognize and affirm the results of EMD by confining the anomalous zones of each IMF.

\subsection{De-noising}

After the generation of synthetic seismic trace with $10 \%$ noise, EMD is applied to the raw seismic trace, which produced five IMFs, as shown in figure 3. In this study, EMD acts as a time-domain filter. During the generation of IMFs, the noise starts to decrease and the seismic reflections are getting prominent. The $1^{\text {st }}$ IMF represents all the high frequencies of the original signal. Then, in the $2^{\text {nd }}$ and $3^{\text {rd }} \mathrm{IMF}$, noise is reduced significantly and the seismic reflections are prominent at $0.8,1$, and $1.07 \mathrm{sec}$. As we move towards the $4^{\text {th }}$ and $5^{\text {th }} \mathrm{IMF}$, although the noise is reduced to zero but the seismic reflections also got affected and the second and third seismic reflections are almost disappeared. This means that $2^{\text {nd }}$ and $3^{\text {rd }}$ IMF could be used as a profound source of information about seismic reflections.

Figure 3: Synthetic seismic trace with $10 \%$ Gaussian noise and its 5-IMFs as a result of EMD

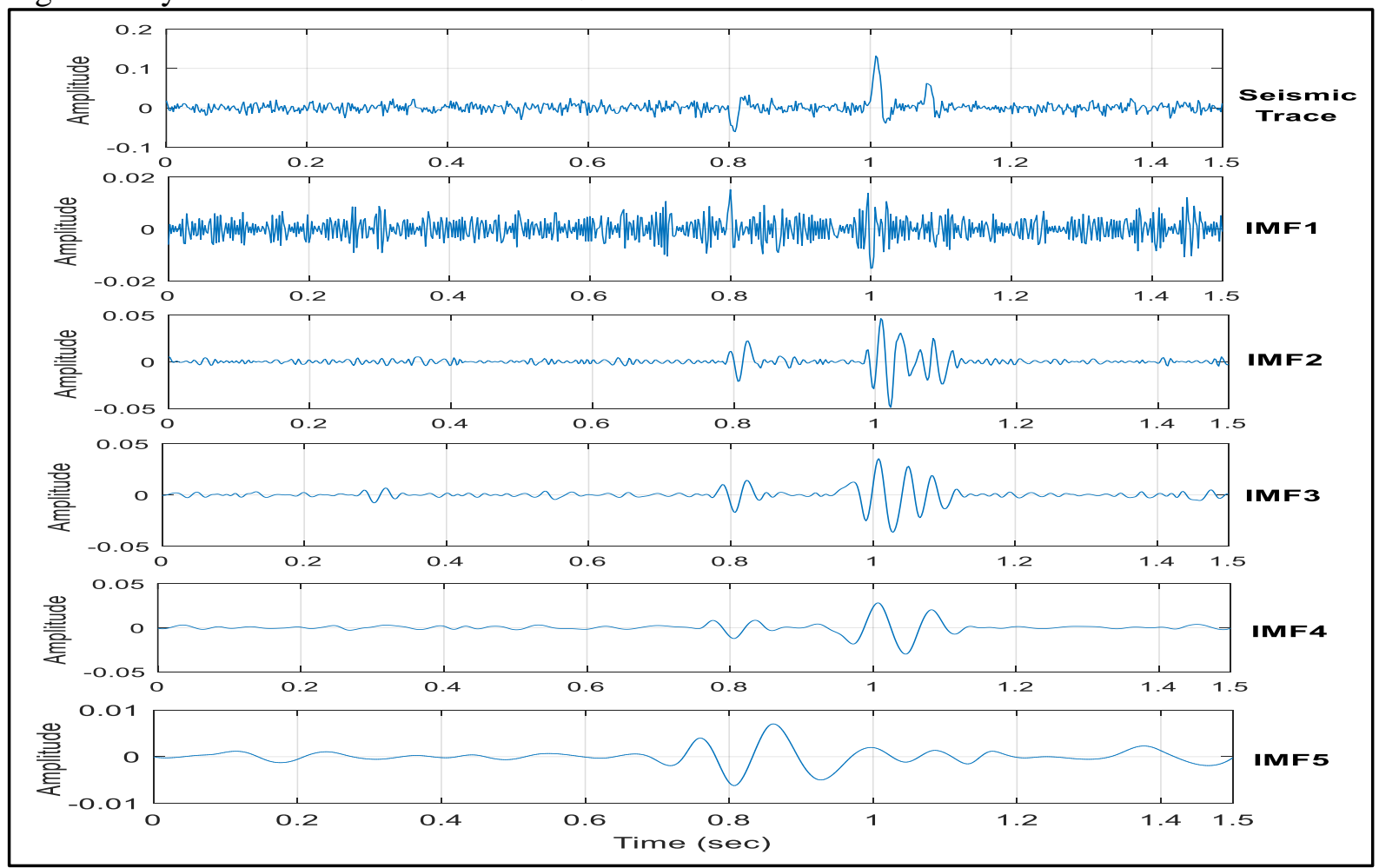

EMD only accounts for high frequencies and strong trends within the time-series signal. Due to the sifting process, it removes the weak and low-frequency trends with the generation of each new IMF. Therefore, during the sifting process, with each new IMF, EMD may miss some weak trends within the signal. Based on the results of EMD, the $2^{\text {nd }}$ and $3^{\text {rd }}$ IMF present the best results to recognize the seismic reflections, and as well as the noise is reduced exceptionally. 


\subsection{Continuous wavelet transformation of IMF}

To select the accurate IMF, each IMF is transformed using CWT, as shown in figure 4-8. For the CWT, the Morlet wavelet is utilized as a mother wavelet for the transformation of each IMF. After EMD implementation on seismic signal, the next, cwt has been adopted on the EMD results to validate the results and properly define the reflection boundaries in the seismic signal.

CWT of each IMF shows that as we move from IMF1 to IMF5, the noise is decreasing significantly, and seismic reflections are getting prominent. Since the $1^{\text {st }}$ IMF contains all the highest frequencies of the signal; therefore, the scalogram of $1^{\text {st }}$ IMF has not shown any prominent seismic reflections as a high energy bright spot. While3D CWT coefficients of $1^{\text {st }}$ IMF exhibits two high energy spectrums at $0.8 \mathrm{sec}$ and $1.0 \mathrm{sec}$ along with some other highfrequency data of the signal, as shown in figure 4.

Figure 4: (a) $1^{\text {st }}$ IMF of seismic trace along with its (b) 2D scalogram and (c) 3D CWT coefficients of IMF

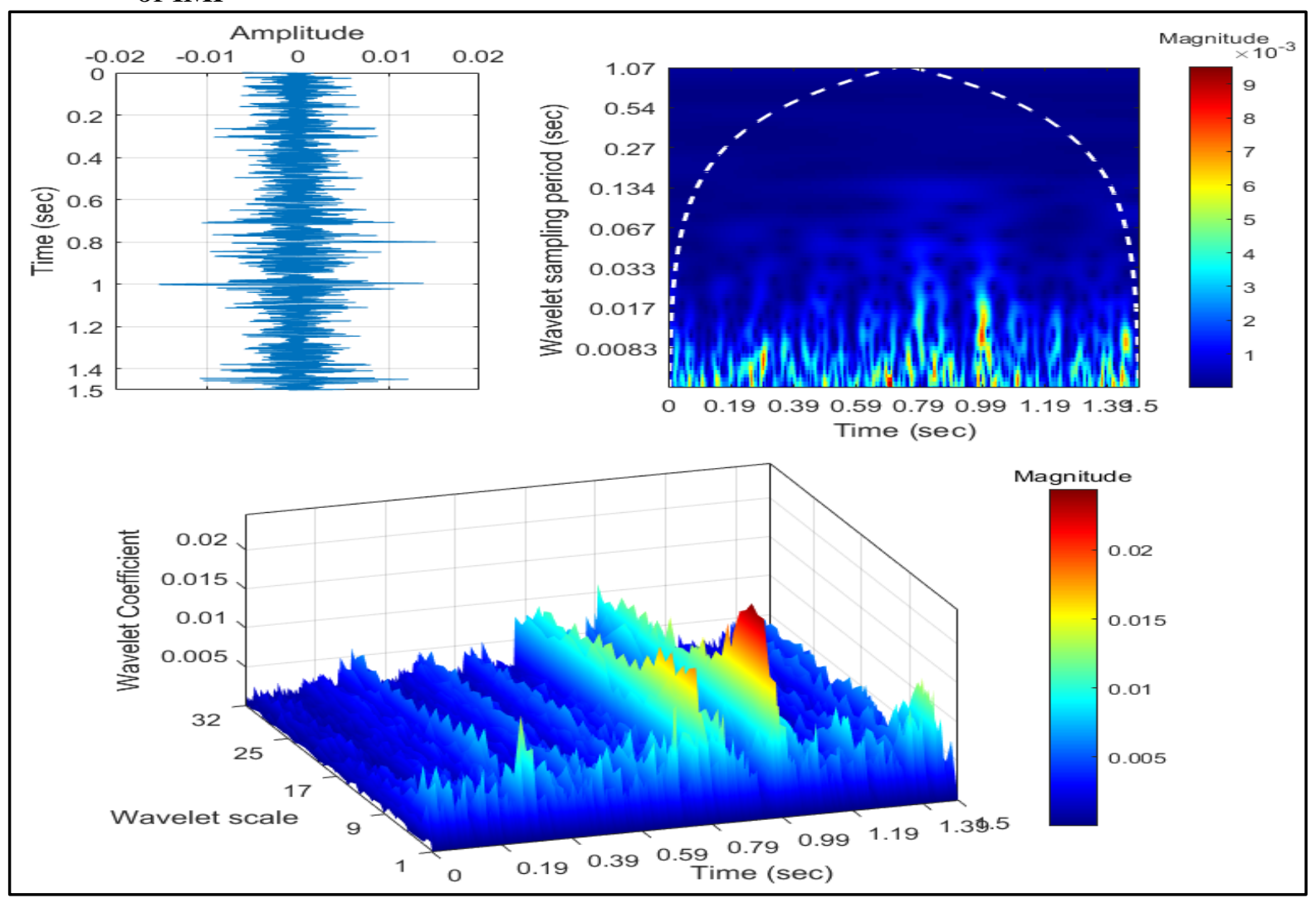

The CWT of $2^{\text {nd }}$ IMF shows that the noise is reduced prominently and the $2 \mathrm{D}$ CWT of $2^{\text {nd }}$ IMF is presented as a scalogram with the cone of influence (COI). Within the COI of $2^{\text {nd }} I M F$, three high energy bright spots are distinguished at $0.8 \mathrm{sec}, 1 \mathrm{sec}$, and $1.07 \mathrm{sec}$. The $1^{\text {st }}$ bright spot is the seismic reflection contact at $0.8 \mathrm{sec}, 2^{\text {nd }}$ bright spot represents the second seismic reflection at $1 \mathrm{sec}$ and the $3^{\text {rd }}$ bright spot is the third seismic reflection at $1.07 \mathrm{sec}$. Similarly, 3D CWT coefficients of the $2^{\text {nd }}$ IMF show much better results in terms of interpretation. Three critical high energy ranges can be easily distinguished at $0.8 \mathrm{sec}, 1 \mathrm{sec}$, and $1.07 \mathrm{sec}$, as shown in the figure 5 . 
Figure 5: (a) $2^{\text {nd }}$ IMF of seismic trace along with its (b) 2D scalogram and (c) 3D CWT coefficients of IMF

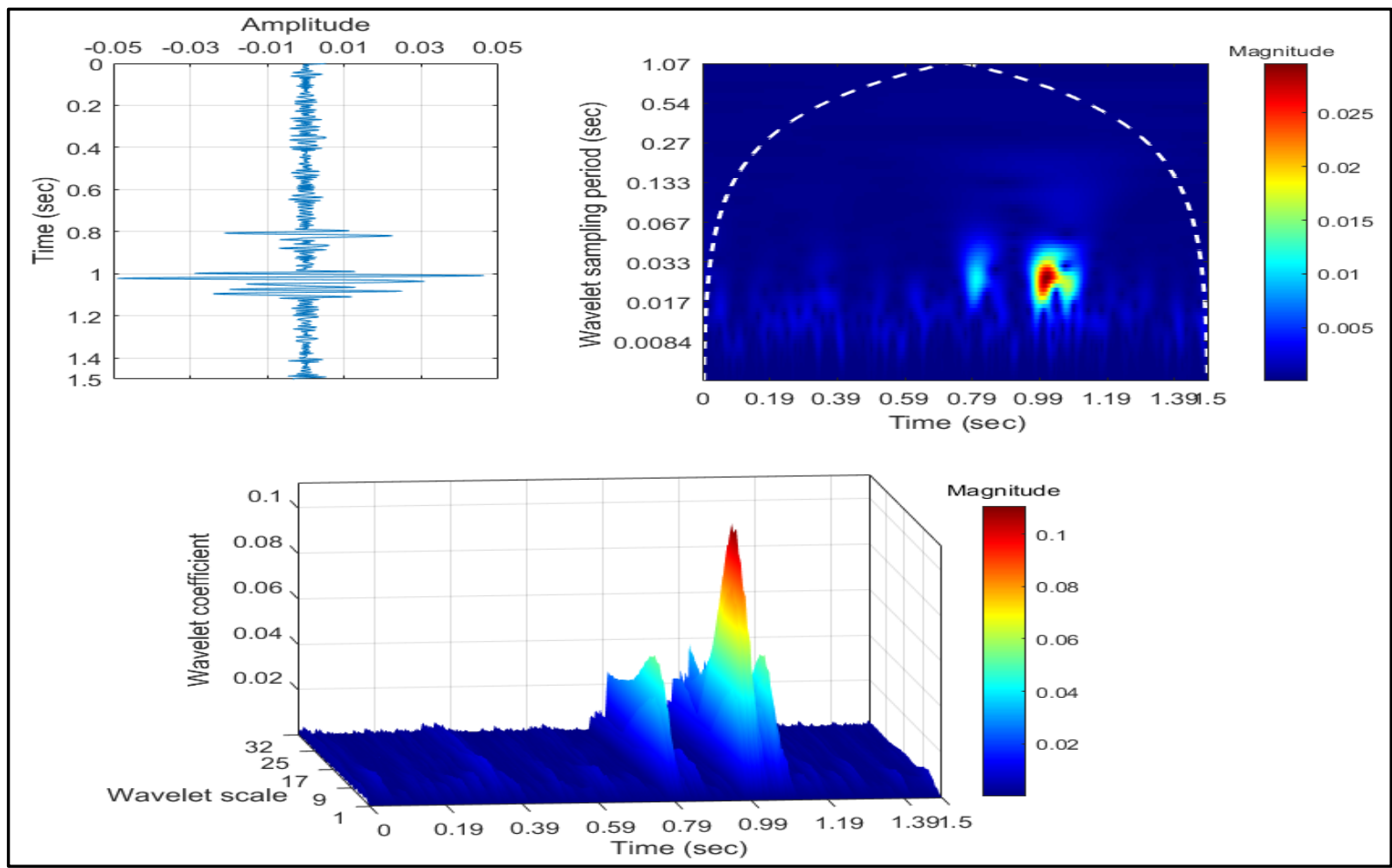

However, as we move towards the $3^{\text {rd }} \mathrm{IMF}, 4^{\text {th }} \mathrm{IMF}$, and $5^{\text {th }} \mathrm{IMF}$, due to the sifting process of EMD, the noise vanished completely, but it also affected the seismic reflections. Because of the weak trend and low frequency, the $3^{\text {rd }}$ reflection is merged with the $2^{\text {nd }}$ reflection, as we can see in the CWT of the last three IMFs, as shown in figures 6-8.

Figure 6: (a) $3^{\text {rd }}$ IMF of seismic trace along with its (b) 2D scalogram and (c) 3D CWT coefficients of IMF

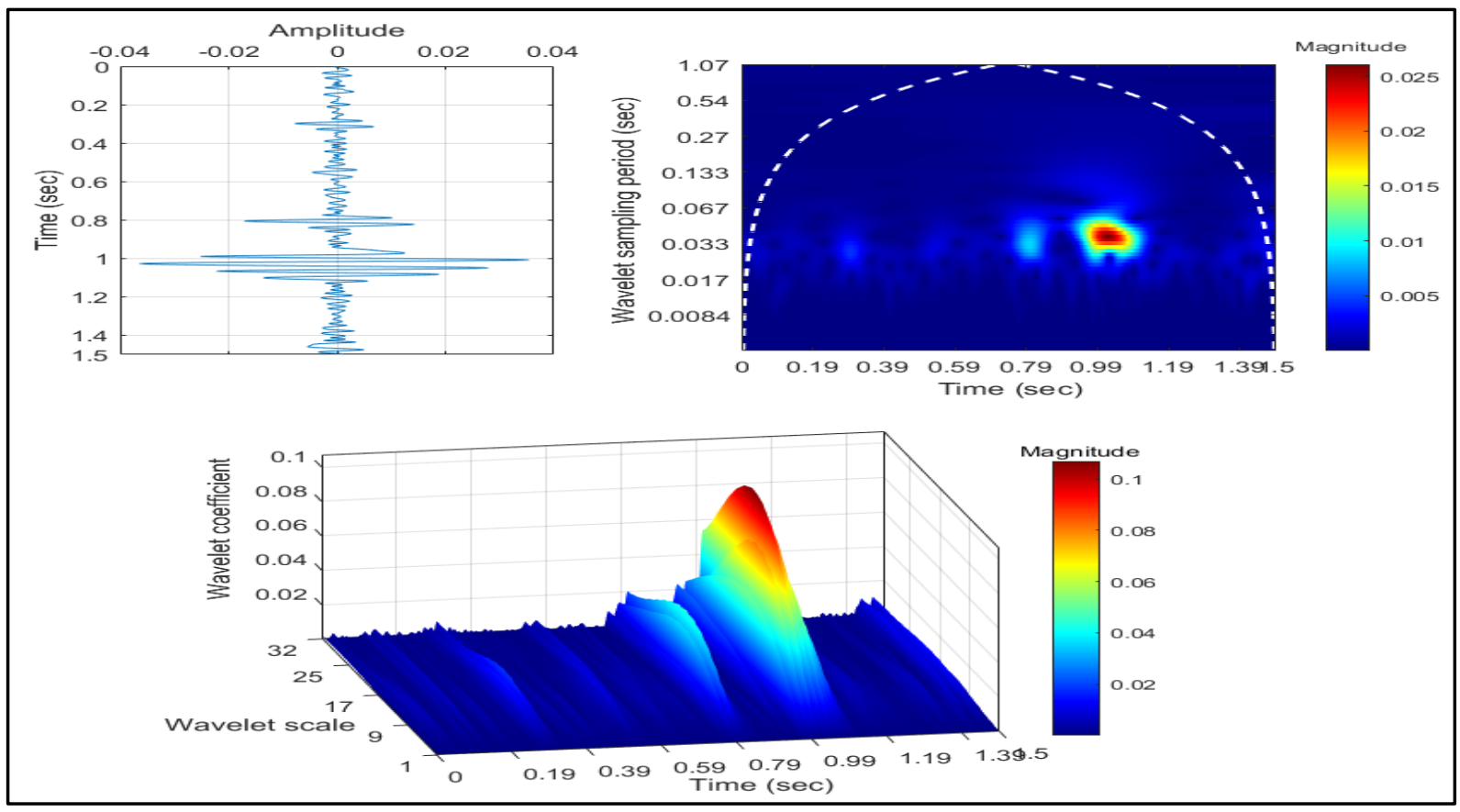


Figure 7: (a) $4^{\text {th }}$ IMF of seismic trace along with its (b) 2D scalogram and (c) 3D CWT coefficients of IMF

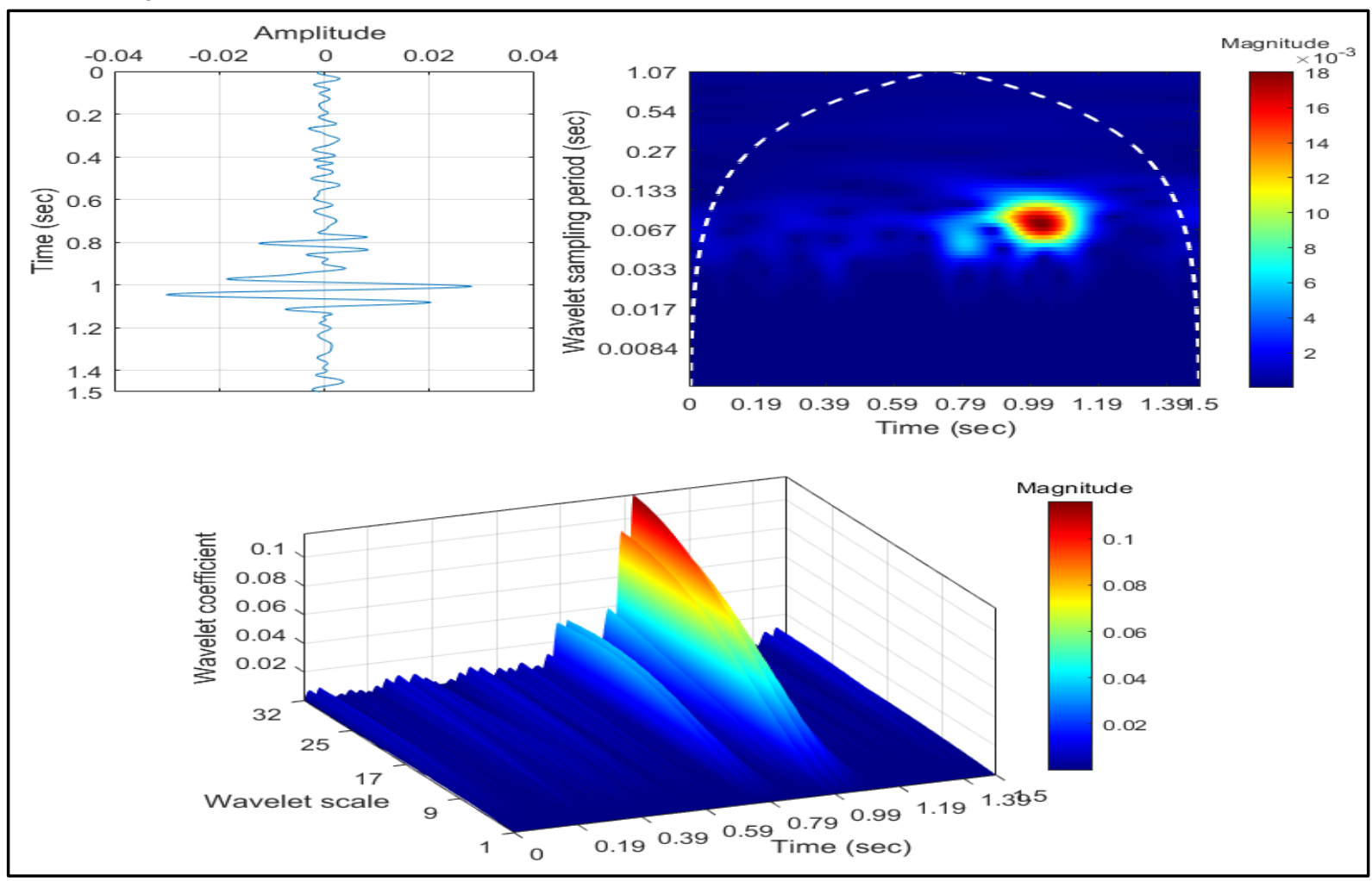

Figure 8: (a) $5^{\text {th }}$ IMF of seismic trace along with its (b) 2D scalogram and (c) 3D CWT coefficients of IMF

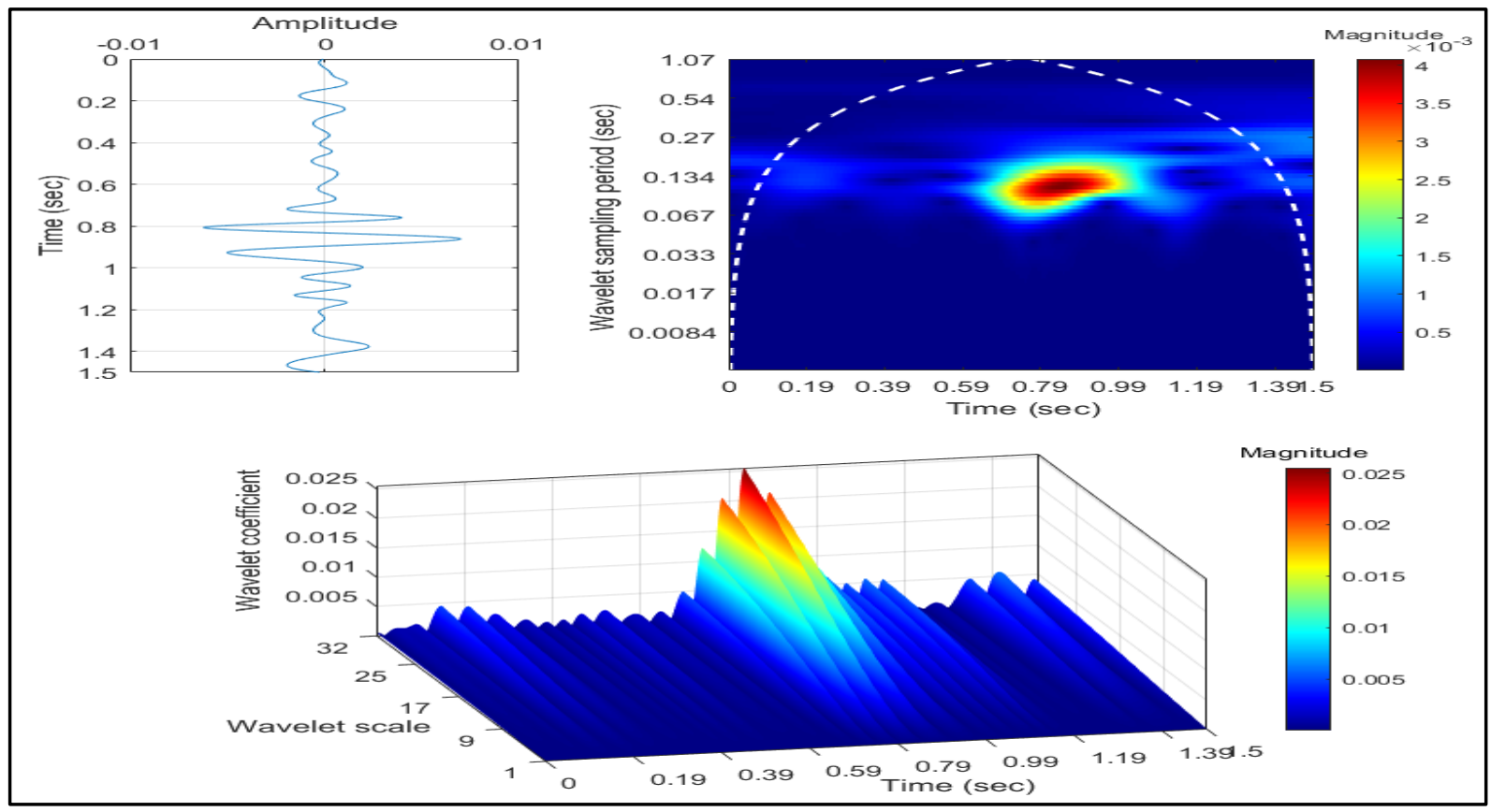

Based on the results of the CWT of each IMF, the best results are given by the $2^{\text {nd }}$ IMF. This suggests that using the EMD as a time-domain filter and CWT as a confirmation tool of recognizing the seismic reflections as a localized anomaly, the $2^{\text {nd }}$ IMF is the best output for further seismic processing and interpretation of seismic reflection data. 
At the end of this study, the statistical analysis is also implemented in terms of confidence interval with a $95 \%$ interval for all five IMFs (figure 9). The sum of all the IMFs is the original signal (Coughlin \& Tung, 2004). The confidence bound also shows the same results, three different points from the sum of all IMFs are found beyond the upper and lower bound as peculiar regions $(0.8 \mathrm{sec}, 1 \mathrm{sec}$, and $1.07 \mathrm{sec})$. This also supports the findings of CWT of IMFs.

Figure 9: Confidence bounds of all IMFs

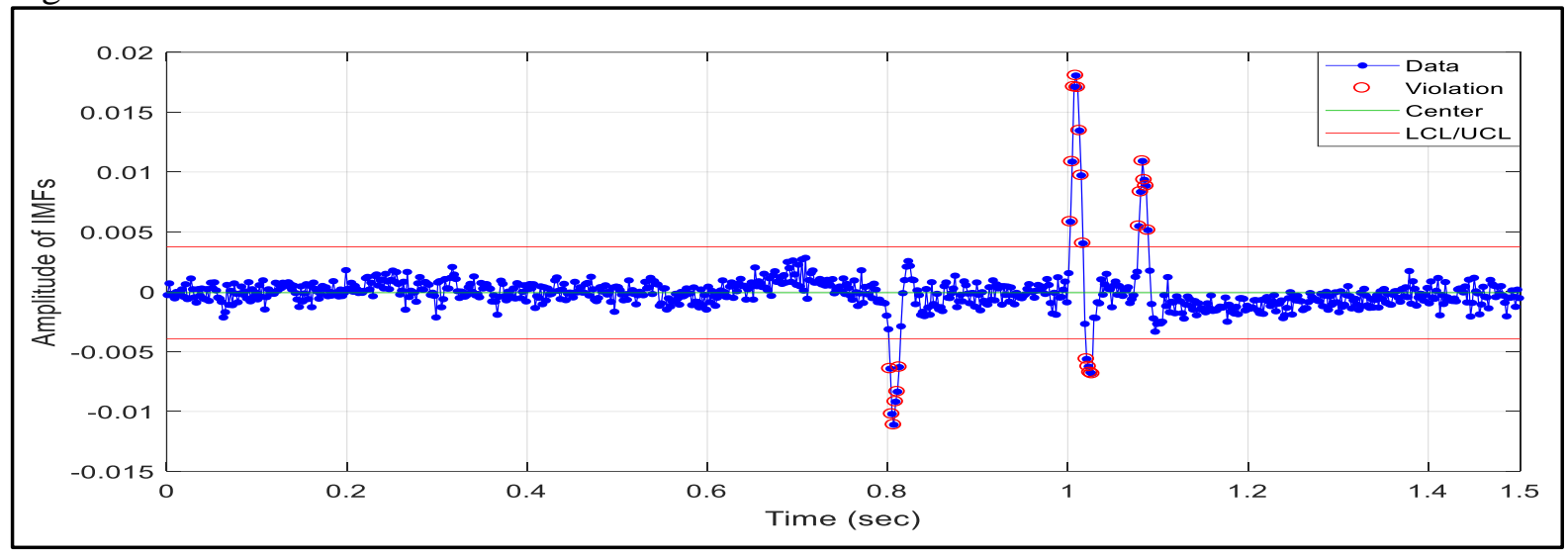

\section{Conclusion}

This study is carried out to examine the non-stationary seismic signal using EMD and CWT. Where EMD has been used as a time-domain filter and CWT is used to localize each reflection at its exact time location. EMD as a time-domain filter remarkably enhanced the signal-tonoise ratio by generating multiple IMFs of the original raw seismic signal. With each new IMF, EMD reduced the noise significantly. The pro of multiple IMFs is that we can choose the best IMF as a true representative of the seismic reflection data. However, the con is that EMD may also remove some weak trends with low frequencies.

CWT can explore a signal within the time and frequency space, simultaneously. In signal processing, the spectral decomposition of a 1D signal into a scalogram and 3D CWT time segment is an extraordinary development. CWT can isolate diverse seismic functions of various seismic frequencies and it can also confine the functions as anomalous regions. CWT fundamentally relies upon the mother wavelet and scaling function.

By implementing EMD and CWT on seismic trace, it gave us better results to verify the actual positions of seismic reflection. These both can be good tools for enhancing the signal-to-noise ratio. Similarly, both can provide deep insights during combined analysis in the interpretation of seismic reflection data.

\section{Acknowledgment}

The authors of this study are grateful to the School of Earth Sciences, Zhejiang University, Hangzhou, China, for providing an excellent working environment and research facilities.

\section{Conflict of interest statement}

On behalf of all authors, the corresponding author states that there is no conflict of interest. 


\section{References}

Adhikari, B. (2015). HILDCAA-related effects recorded in middle-low latitude magnetometers. http://urlib.net/sid.inpe.br/mtc-m21b/2015/02.27.16.58

Batzle, M., \& Wang, Z. (1992). Seismic properties of pore fluids.Geophysics, 57(11), 13961408. https://doi.org/10.1190/1.1443207

Castagna, J. P., Sun, S., \& Siegfried, R. W. (2003). Instantaneous spectral analysis: Detection of low-frequency shadows associated with hydrocarbons. The Leading Edge, 22(2), 120-127. https://www2.lbl.gov/TT/publications/1663pub5.pdf

Cohen, L. (1995). Time-frequency analysis (Vol. 778). Prentice Hall.

Coughlin, K., \& Tung, K.-K.(2004). Eleven-year solar cycle signal throughout the lower atmosphere.Journal of Geophysical Research: Atmospheres, 109(D21). https://doi.org/10.1029/2004JD004873

Flandrin, P., Rilling, G., \&Goncalves, P. (2004). Empirical mode decomposition as a filter bank. IEEE Signal Processing Letters, 11(2), 112-114. https://doi.org/10.1109/LSP.2003.821662

Gabor, D. (1946). Theory of communication. Part 1: The analysis of information. Journal of the Institution of Electrical Engineers-Part III: Radio and Communication Engineering, 93(26), 429-441.

Holschneider, M. (1995). Wavelets an analysis tool.

Huang, N. E., Shen, Z., Long, S. R., Wu, M. C., Shih, H. H., Zheng, Q., Liu, H. H. (1998). The empirical mode decomposition and the Hilbert spectrum for nonlinear and nonstationary time series analysis. Proceedings of the Royal Society of London. Series A: Mathematical, Physical and Engineering Sciences, 454(1971), 903-995. https://doi.org/10.1098/rspa.1998.0193

Klausner, V., Papa, A. R., Mendes, O., Domingues, M. O., \& Frick, P. (2013). Characteristics of solar diurnal variations: A case study based on records from the ground magnetic station at Vassouras, Brazil. Journal of Atmospheric and Solar-Terrestrial Physics, 92, 124-136. https://doi.org/10.1016/j.jastp.2012.10.007

Iqbal, I., Tian, G., Iqbal, S., \& Rahman, N. (2018a). Analysis of influence of AVO on Murree and Sakesar formations in upper Indus basin Pakistan. Geologica Acta, 3(1).

Iqbal, I., Tian, G., Khan, A., \& Iqbal, S. (2018b). Integrated geophysical analysis and rock physics study to confirm the hydrocarbon reservoir of the Bitrisim area in Pakistan. Journal of Geology \& Geophysics, 7(2), 1000330. https://doi.org/10.4172/2381$\underline{8719.1000330}$

Liu, S., Zhou, Z., Dai, S., Iqbal, I., \& Yang, Y. (2021). Fast computation of green function for layered Seismic field via Discrete Complex Image Method and Double Exponential Rules. Symmetry, 13(10), 1969. https://doi.org/10.3390/sym13101969

Magrin-Chagnolleau, I., \&Baraniuk, R. G. (1999). Empirical mode decomposition based timefrequency attributes. In SEG Technical Program Expanded Abstracts 1999 (pp. 19491952): Society of Exploration Geophysicists.

Mallat, S. G. (1987). A theory for multiresolution signal decomposition: The wavelet representation.

Partyka, G., Gridley, J., \& Lopez, J. (1999). Interpretational applications of spectral decomposition in reservoir characterization. The Leading Edge, 18(3), 353-360.

Peyton, L., Bottjer, R., \&Partyka, G. (1998). Interpretation of incised valleys using new 3-D seismic techniques: A case history using spectral decomposition and coherency. The Leading Edge, 17(9), 1294-1298. 
Palupi, I. R. (2018). Depth prediction of gravity data by using continuous wavelet transform. Paper presented at the EAGE-HAGI 1st Asia Pacific Meeting on Near Surface Geoscience and Engineering.

Rilling, G., Flandrin, P., \&Goncalves, P. (2004).Detrending and denoising with empirical mode decomposition. Paper Presented at the Proceedings of EUSIPCO (pp. 1581-1584).

Rioul, O., \& Vetterli, M. (1991). Wavelets and signal processing. IEEE Signal Processing Magazine, 8(4), 14-38.https://doi.org/10.1109/79.91217

Torrence, C., \& Compo, G. P. (1998). A practical guide to wavelet analysis. Bulletin of the American Meteorological Society, 79(1), 61-78.https://doi.org/10.1175/15200477(1998)079\%3C0061:APGTWA\%3E2.0.CO;2

Wu, Z., \& Huang, N. E. (2004). A study of the characteristics of white noise using the empirical mode decomposition method. Proceedings of the Royal Society of London. Series A: Mathematical, Physical and Engineering Sciences, 460(2046), 15971611.https://doi.org/10.1098/rspa.2003.1221

Yilmaz. O. (2001). Fundamentals of signal processing Seismic Data Analysis (pp. 25-158): Society of Exploration Geophysicists.

Zhou, Z. J., \& Chen, J. (2020). Singularity detection based on two-dimensional continuous wavelet transform. Journal of Image Signal Processing, 9, 111-118. 